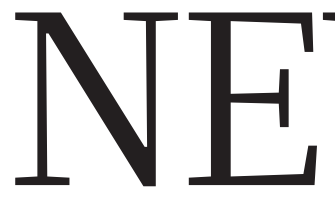

BIOMEDICINE Cancer researchers push to relax criteria for inclusion in clinical trials p.12
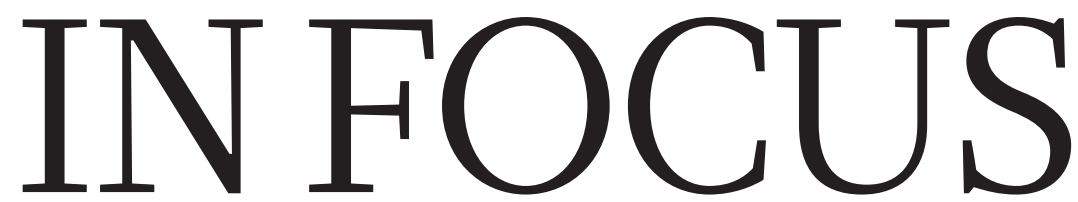

ASTROPHYSICS Upgraded Italian detector spots signs of elusive dark matter p.13
PUblishing Potential

changes to EU copyright rules rile researchers $\mathbf{p . 1 4}$

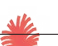

Immunology Could dirtier

1 lab mice make for better science? p.16

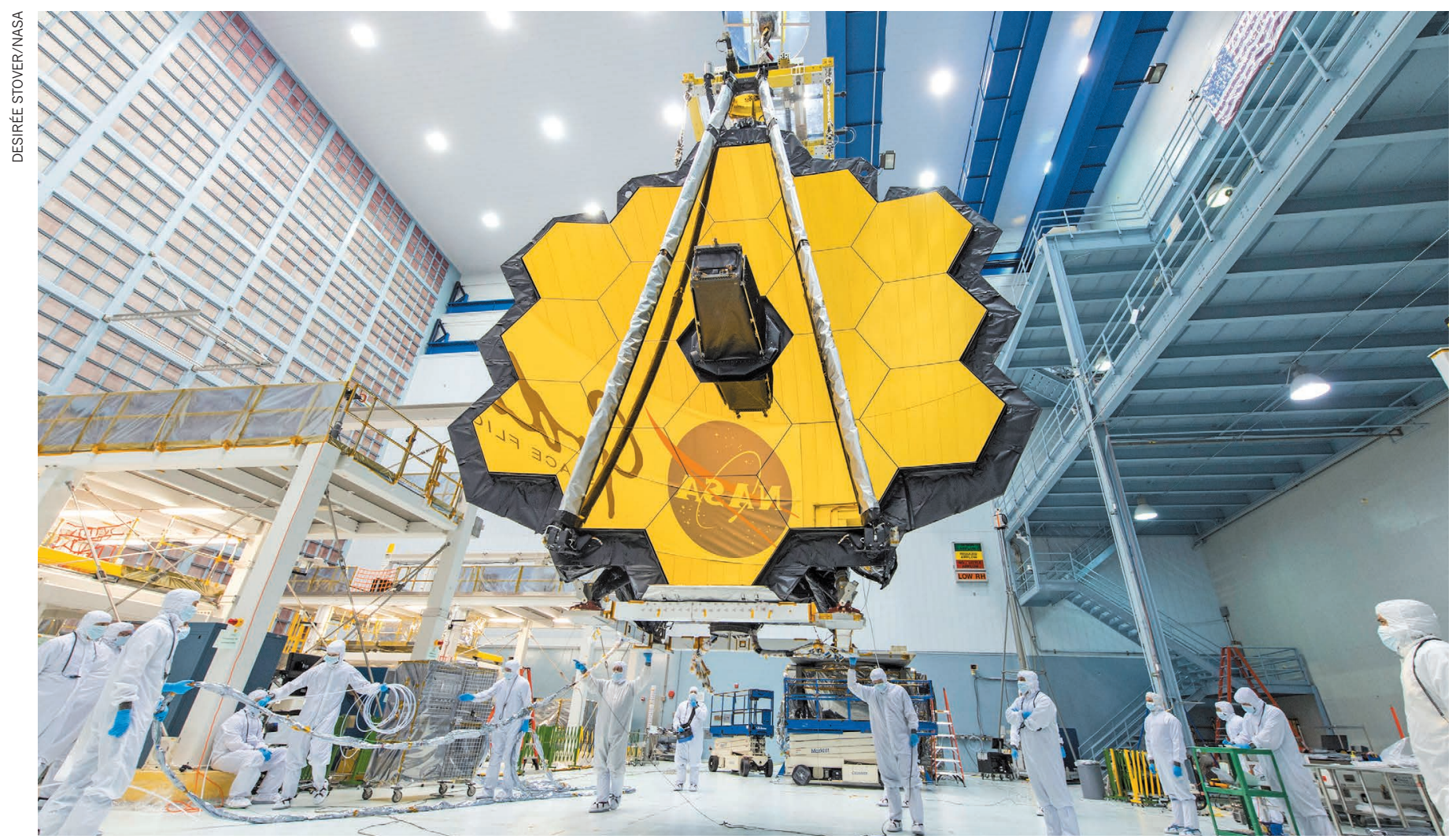

The mirrors of the James Webb Space Telescope are designed to peer at the Universe's first stars.

\title{
NASA reveals major delay for Hubble successor
}

\section{James Webb Space Telescope woes could have broader effects on astrophysics programme.}

\section{BY ALEXANDRA WITZE}

$\mathrm{N}$ ASA will delay the launch of its ambitious James Webb Space Telescope (JWST) by nearly a year, until approximately May 2020. That is likely to push the cost of the mission - the most complex space-science telescope ever built - over the US\$8-billion limit set by the US Congress. It is the first major setback since NASA revised its plans for the project in 2011, after years of slipping schedules and rising costs.

NASA announced the delay on $27 \mathrm{March}$, saying that engineers needed more time to assemble and test the components of the spacecraft at its main contractor, Northrop Grumman in Redondo Beach, California. Among other problems, the collapsible, tennis-court-sized sunshield that will protect the observatory's 6.5-metre mirror took weeks longer than expected to fold and refold during testing. "Frankly, the tests are taking longer to complete than expected," Robert Lightfoot, NASA's acting administrator, told reporters.

The agency did not say how much the delay would cost, but some estimates suggest that it could add a few hundred million dollars to the project. In recent years, Congress has pushed NASA to hold down the cost of the telescope and other future missions.

The delay will affect NASA's astrophysics budget more broadly, including its next big planned space observatory, the Wide-Field 
- Infrared Survey Telescope (WFIRST). The agency had aimed to spend more on WFIRST in the coming years as its contributions to the JWST shrank, trading off the end of one mission's development for the beginning of another. Now, the JWST delay risks compounding problems for WFIRST. Last month, US President Donald Trump proposed cancelling WFIRST; astronomers protested, and Congress gave the project $\$ 150$ million.

US astronomers ranked the JWST and WFIRST as the most important large space missions, respectively, in decadal surveys of their scientific priorities released in 2000 and 2010.

NASA delayed the JWST launch after an independent board of experts concluded this month that the project could not meet its June 2019 launch goal. Last September, the agency shifted to that target, abandoning the October 2018 launch date it had aimed for since rebooting the project in 2011.

Now, yet another independent panel — led by former aerospace executive Tom Young will review the project's schedule. NASA will use these analyses to pick a more specific launch target in the coming months. "We have one shot to get this right before going into space," says Thomas Zurbuchen, NASA's associate administrator for science.

Until recently, project managers were able to deal with schedule problems by moving work tasks around locations. For instance, engineers decided to clean the telescope's mirror at NASA's Johnson Space Center in Houston before shipping it to the crowded spacecraftassembly facility at Northrop.

Northrop staff are now working 24 hours a day, but cannot handle enough parts simultaneously to stay on schedule. The increased number of workers is adding to the project's

"We have one
shot to get this
right before
going into
space."
overall cost, the US Government Accountability Office reported last month.

There are other problems, too. In April 2017, a technician applied too high a voltage during a test, damaging components of the propulsion system that took more than a month to replace. In October, engineers discovered several tears in the sunshield caused by "workmanship error". And part of the five-layer sunshield snagged during a deployment test.

In a statement, Northrop said that it "remains steadfast in its commitment to NASA and ensuring successful integration, launch and deployment" of the telescope.

All the testing is crucial because the JWST will operate from an orbit about 1.5 million kilometres from Earth, where it cannot be serviced by astronauts as the Hubble Space
Telescope was. The JWST will be 100 times more powerful than Hubble, and will survey the Universe mainly in infrared wavelengths. Among the many celestial phenomena that it aims to explore are the first stars and galaxies to form in the Universe, as well as planets in and beyond the Solar System.

NASA had asked scientists to submit proposals for the JWST's first set of observations by next week, but the agency has cancelled that deadline. "We'd rather have it launch later and work perfectly than rush and have problems," says Emily Levesque, an astronomer at the University of Washington in Seattle. "But there are going to be a lot of people considering what this means for astronomy."

Garth Illingworth, an astronomer at the University of California, Santa Cruz, says that the next decadal survey, scheduled for 2020, should be postponed. "It's hard to imagine a group of people thinking clearly about what to do in the future with uncertainty about JWST's performance hanging around," he says.

NASA will also have to figure out how to accommodate the extra costs - perhaps by taking them out of the operations budget for the JWST, penalizing Northrop or delaying WFIRST. The agency has spent $\$ 7.3$ billion on the JWST so far, Lightfoot said, and cannot exceed $\$ 8$ billion without permission from Congress.

\section{BY HEIDI LEDFORD}

$\mathrm{N}$ early $20 \%$ of publicly funded cancer clinical trials in the United States fail because investigators are unable to enrol enough participants. Yet patients and their physicians often grow frustrated when they encounter the sometimesinsurmountable requirements to join a study.

Now, researchers are pruning the lengthy lists of eligibility criteria for trials, in the hope of nixing unnecessary rules that might be hindering research. On 16 April, representatives of the US Food and Drug Administration (FDA) will meet stakeholders in Washington DC to discuss how restrictive eligibility criteria for clinical trials could be limiting people's opportunities to access experimental treatments and the quality of the data generated by the studies. The agency plans to use the information it gathers to develop guidelines for drug makers.

"You can have the greatest ideas and the greatest science," says Stuart Lichtman, an oncologist at Memorial Sloan Kettering Cancer Center in New York City. "But if no one goes on the study, what good is it?"

Eligibility requirements are typically intended to protect either the participant or the study. Participants with some degree of liver failure, for example, might not be allowed to take part in a trial of a drug thought to pose a risk to that organ. Criteria might also exclude people with conditions that could confound the results of a study.

But some researchers say that a 'cut-andpaste' mentality has increased clinical-trial requirements over time, as scientists have used previous trial protocols as templates for their next studies. That might be needlessly restricting participation in trials.

David Gerber, a lung-cancer specialist at the University of Texas Southwestern Medical Center in Dallas, and his collaborators have found that $80 \%$ of clinical trials sponsored by the US National Cancer Institute excluded people with previous cancer diagnoses (D. E. Gerber et al. J. Natl Cancer Inst. 106, dju302; 2014). Yet in many cases, he says, the previous cancer might have been caught early and removed successfully before the person developed lung cancer.

"What really frustrates me are instances when, in my mind and in my heart, it really seemed that the patients should be eligible," says Gerber. "If I had the exact same treatment outside of a clinical trial, I would give 